\title{
De prácticas expulsivas a construcciones colectivas: relato de experiencias de transversalidades en clave de discapacidad / accesibilidad en la UNLP
}

\author{
From discriminatory practices to collective constructions: a report \\ on experiences of transverse paths in connection with disabilities / \\ accessibility at the UNLP
}

\section{Sandra L. Katz}

https://orcid.org/0000-0001-8110-9697

sandrakatz4@gmail.com

Universidad Nacional de La Plata | Argentina

\section{Lorena Miranda}

https://orcid.org/0000-0003-2510-8475

lorena.miranda@gmail.com

Universidad Nacional de La Plata | Argentina

\section{RESUMEN}

Desde la Dirección de Inclusión, Discapacidad y DDHH de la Universidad Nacional de La Plata (UNLP), compartimos en el presente relato, acciones realizadas en tiempos de pandemia en pos de promover y garantizar los derechos de las personas con discapacidad desde una perspectiva de accesibilidad: Una de las capacitaciones llevadas a cabo para la producción de accesibilidad académica, y el desarrollo de una aplicación accesible para celulares. Dichas acciones están dirigidas a estudiantes, docentes, nodocentes, graduados y comunidad en general, con el objetivo de ofrecer recursos que garanticen el derecho a la educación. Entendemos que la discapacidad es una construcción social donde, desde una mirada inclusiva en coherencia con lo establecido por el estatuto de la UNLP, las normativas nacionales e internacionales, se pone en relieve el trabajo desde un enfoque transversal, interdisciplinario y colectivo. En la medida que se construyan políticas institucionales en clave de accesibilidad se está garantizando que cualquier persona con discapacidad pueda sentirse alojado por UNLP.

PALABRAS CLAVE

Discapacidad, Accesibilidad, Apoyos, Educación superior. 
KEY WORDS

Disability;

Accessibility;

Supports,

Higher Education.

\section{ABSTRACT}

From the Department of Inclusion, Disability and Human Rights of the UNLP, we hereby share in the present report the actions taken during the pandemic to promote and guarantee the rights of people with disabilities, from a perspective of accessibility. These aforementioned actions include a training course, which was carried out by the production of academic accessibility, and the development of an app for mobile phones. Such actions are directed towards students, educators, non-educators, graduates and the community in general, to offer resources that guarantee the right to education. We understand that disability is a social construction in which - from an inclusive perspective in terms of what has been established by the UNLP statute, along with the national and international normative - the accomplished work is seen from a transversal, interdisciplinary and collective perspective. Upon formulating institutional policies regarding accessibility, it is guaranteed that any person with a disability shall feel welcome to UNLP. 
Desde el año 2000, cuando se creó la Comisión Universitaria sobre Discapacidad (CUD) se desarrollan acciones en pos de visibilizar y generar políticas en clave de accesibilidad, y fue en el año 2016, cuando como Dirección de Inclusión, Discapacidad y DDHH (en adelante la Dirección) pasa a formar parte de una de las cinco áreas de la Prosecretaría de DDHH de la UNLP (https://unlp.edu.ar/ddhh), permitiendo anclar de esta manera el encuadre necesario para institucionalizar políticas

En estos intensos y maravillosos años de trabajo fuimos aprendiendo a llevar la mirada hacia aquel aspecto en el que había (o debíamos) hacer foco. Un aprendizaje colaborativo, un hacer haciendo a partir de estar atentos y atentas a la demanda y a la urgencia. Estaba todo por hacer. Un tema invisibilizado, casi que no era un problema, no estaba en la agenda, no había reclamos, apenas se conocía de manera aislada alguna denuncia, pero no había pedidos para que intervengamos. Sin embargo, una vez que comenzó a ponerse en palabras, se transformó en torbellino de ideas, demandas, diversificaciones, barreras, resistencias y sueños.

Hoy somos un equipo de trabajo interdisciplinario, coordinado por Sandra Katz (Psicóloga y Prof. de Educación Física) y conformado por Evelina Diaz (Trabajadora Social), Maria Innaro (Diseñadora gráfica), Lorena Miranda (Bibliotecaria), Gonzalo Ithurbide (Multimedia), cinco Intérpretes de Lengua de Señas Argentina - Español (ILSA-E) : Katterine Jarandilla; Silvina Monteros Abregú; Laura Meza, Florencia Espinal y Paula Salas, y la valiosa y necesaria participación de asesores con discapacidad: Joana Sarmiento en relación a las personas ciegas y Juan Manuel Barbato para la Comunidad Sorda.

Podríamos retomar esa construcción e historización, pero, ante la convocatoria a escribir este artículo, decidimos compartir dos actividades que fueron implementadas en el marco de la pandemia: capacitaciones virtuales para la elaboración y producción de materiales en formatos accesibles y una aplicación para el celular que dé información en formato accesible a la comunidad universitaria.

Tal vez, en algunos ámbitos se piense que la discapacidad es un problema que tienen algunas personas, y se podría ampliar a algunas 
familias. Pero, posicionados desde el modelo social, entendemos que los "problemas o barreras" que se encuentran las Personas con Discapacidad (PCD) son generados por la misma sociedad, y cuando estamos atravesados por la "ideología de la normalidad" (Angelino, 2009), parecería que hay una sola forma válida y legítima de transitar la vida universitaria. Entendemos a la discapacidad como una construcción social, política y cultural que va más allá de las corporalidades, siendo que la misma se construye y reproduce bajo lógicas capacitistas que ponen bajo sospecha a esas corporalidades que rompen con lo deseable y esperable. En línea con lo antedicho, es de relevancia el marco legal vigente aportado por la Convención sobre los Derechos de las Personas con Discapacidad (CDPD, 2006), la cual pone énfasis en valores como la participación, accesibilidad, autonomía, vida independiente y educación inclusiva, siendo estos aspectos principales al planificar, evaluar y ejecutar políticas.

\section{Entendemos a la discapacidad como una construcción so- cial, política y cultural que va más allá de las corporalidades, siendo que la misma se construye y reproduce bajo lógicas capacitistas que ponen bajo sospecha a esas corporalidades que rompen con lo deseable y esperable.}

Las PcD no eligen su condición: culminaron sus estudios, llegan a la universidad, y una vez que comienzan a soñar y proyectarse en un futuro, el cual se les fue arrebatado por mucho tiempo como destino posible, encuentran barreras, prejuicios, miradas despectivas y prácticas excluyentes. Ahí comprendemos, que el problema lo tiene la institución y sus habitantes: docentes, trabajadores, compañeros y compañeras gestores, hacedores de políticas, entre otres, que sin pensarlo ni darse cuenta, construyen un formato en sus prácticas que no puede ser usado por PcD, ya que no son accesibles.

Entonces, desde la Dirección no sólo tuvimos que hacer foco en acompañar a los y las estudiantes en situación de discapacidad que ingresaban (o que después de varios años de esfuerzo individual -y por lo general acompañado por la familia- decidieron dar a conocer su situación y solicitar apoyos), sino también ofrecer acompañamiento a docentes, nodocentes, agrupaciones, etc. para orientar sobre cómo hacer sus prácticas accesibles.

En contexto de pandemia, algunas cuestiones no escapan de la generalidad: un porcentaje importante de estudiantes abandonaron sus estudios, al no poder sostener las cursadas desde la virtualidad, tanto porque preferían esperar a la presencialidad, tuvieron que priorizar lo económico o porque no contaban con dispositivos que les permita co- 
nectarse (Krause, 2021). A esta generalidad se le sumó, en nuestro caso, que algunos estudiantes que sí pudieron sostener el deseo, tenían resuelta la situación económica y los dispositivos necesarios, pero al momento de conectarse el formato que ofrecía el docente no era accesible y quedaba afuera. Ante esta situación redireccionamos nuestras acciones generando diferentes estrategias de capacitación y vías de información donde se pudiera consultar para conocer, implementar y/o revisar las formas de producir conocimiento para que sean accesibles.

Lo primero que generamos fue un blog: https://blogs.ead.unlp.edu. ar/discapacidadunlp/ donde elaboramos materiales sencillos, de lectura amigable con información vinculada a discapacidad y accesibilidad, tanto propia como compilando materiales de nuestras redes: la Red Interuniversitaria de Discapacidad (RID-CIN) http://rid.cin.edu.ar/, y la Red Interuniversitaria Latinoamericana y del Caribe sobre Discapacidad y DDHH http://red-universidadydiscapacidad.org/. Entre otros documentos, en el blog se pueden encontrar tres series: "Consideraciones dialogadas", donde vamos trabajando sobre algunas situaciones discapacitantes, junto a los y las protagonistas, explicando qué es necesario saber para la vida universitaria; ¿Cómo hago?" donde a través de tutoriales se explican algunas cuestiones vinculadas a accesibilidad en la virtualidad; $y$ "Capacitaciones virtuales" donde se comparte el material de las capacitaciones impartidas.

Al mismo tiempo, en sincronía con diferentes espacios de la UNLP que le hacían frente a las urgencias de la no presencialidad, fuimos convocados por la Dirección de Educación a Distancia, para realizar un Webinar que se llamó "Accesibilidad y discapacidad" que nos permitió abrir nuevos diálogos con docentes que tenían en sus cátedras estudiantes con discapacidad: https://youtu.be/3BneGe8owv0, dicho video a un año y medio de haberlo realizado tiene 1608 visualizaciones.

De la serie Capacitaciones Virtuales Ilevadas a cabo, elegimos relatar la de "Producción de videos accesibles". y el otro relato, es el diseño de la Aplicación que nos permite ofrecer acompañamiento a estudiantes con discapacidad, dado que nos dieron a conocer las dificultades que encontraban para localizar información vinculada a los apoyos, referentes, espacios de la universidad en formato accesible

Creemos necesario aclarar que, aunque nuestra aspiración es lograr que en algún momento no sean necesarias estas políticas focalizadas, hoy es la única opción.

\section{CAPACITACIÓN VIRTUAL PARA OFRECER CONDICIONES DE ACCE- SIBILIDAD ANTE LA VIRTUALIDAD.}

(Relato realizado con el aporte de Gonzalo Ithurbide)

A partir marzo del 2020, dado el contexto de pandemia debido al COVID-19, se tuvieron que adecuar las formas de dar clases a través de 
la virtualidad, lo que requirió entender el tipo de producción audiovisual necesaria para la población sorda universitaria, usuaria de lengua de señas. Para abordar esta demanda, estuvimos en diálogo permanente con estudiantes sordes, conociendo cuáles eran las necesidades que les permitieran participar de las clases virtuales, ya sean sincrónicas o asincrónicas. El principal obstáculo estaba dado en la forma de incorporar la figura de la ILSA-E. En el caso de las clases sincrónicas se requería fijar la imagen de las intérpretes de alguna manera que no perdiese fidelidad en su expresión. Y en las clases asincrónicas, incorporar a posteriori la ILSA-E.

A medida que pasaban los meses y la situación de cuarentena se extendía, las clases continuaban brindándose de manera virtual, y los distintos referentes institucionales iban sumando consultas, inquietudes, pedidos, por parte de los equipos docentes, para poder solucionar los inconvenientes que se generaban a través de la virtualidad: problemas de sonido, de mala imagen, con la calidad de las producciones, subtitulado, desconocimiento de cómo incorporar la ILSA-E y el tamaño para que esté siempre visible, etc.

Todas estas consultas, fueron dando origen a la capacitación, con la idea de poder brindar herramientas simples, permitiendo generar la capacidad de instalar de manera institucional, una lógica de producción audiovisual en cada Unidad Académica y puedan adecuarlo según sus necesidades al momento de producir sus materiales.

Los temas tratados fueron: cómo editar los videos, edición de sonido; incorporación de texto a modo de subtitulado; incorporación de la imagen de la ILSA-E; la importancia de la audiodescripción; conocimiento de plataformas para difusión de videos; incorporación y edición del subtitulado automático.

\section{Los temas tratados fueron: cómo editar los videos, edición de sonido; incorporación de texto a modo de subtitulado; incorporación de la imagen de la ILSA-E; la importancia de la audiodescripción; conocimiento de plataformas para di- fusión de videos; incorporación y edición del subtitulado automático.}

En la Primera Capacitación Virtual Asincrónica de Producción de Videos Accesibles, se realizó la convocatoria a través de mails institucionales, redes sociales y grupos de WhatsApp. En 48 hs, se recibieron 183 inscripciones. Dada la cantidad de personas interesadas, se resolvió priorizar a quienes tengan relación directa con la UNLP, siendo éstas 116. Así surge una segunda Capacitación, donde se tuvieron en cuenta las 67 personas pendientes de la primera convocatoria, y además se adecuaron algunos temas, lo que llevó a profundizar sobre la audiodes- 
cripción, incorporación del subtitulado e imágenes.

Debido a la amplia difusión en las redes sociales y blog oficial de la Dirección, muchas personas sin relación con la UNLP, solicitaban, desde sus espacios institucionales y personales, ser parte de alguna de estas capacitaciones. La mayor demanda provenía del interior del país y eran personas vinculadas con la temática de discapacidad / accesibilidad. Así surgió la tercera capacitación, en la que participaron docentes de niveles secundarios, terciarios y universitarios de distintas regiones del país. Hubo 121 inscripctos provenientes de distintos municipios de la Pcia. de Bs As; de localidades de la Pcia. de Santa Fé; de la Pcia. de San Luis y de Río Negro.

El $80 \%$ de las personas interesadas eran docentes, el 12\% formaban parte de equipos de orientación, y el $8 \%$ de la comunidad en general.

Al consultarles sobre sus espacios de trabajo, el $62 \%$ refiere que pertenecen a escuelas e Institutos de Educación Especial; el 19\% tiene relación con espacios con situaciones de discapacidad; y el 19\% expresó ser estudiante, trabajar en espacios de gobierno, universidades y público general.

Sobre la plataforma elegida para brindar las capacitaciones, se optó por Classroom ya que cumple con estándares de accesibilidad para lectores de pantalla. Se realizaron 22 videos tutoriales, en los que se explicó de manera didáctica y audiovisual cada una de las aplicaciones que actualmente se usan desde la Dirección, para la producción de videos accesibles.

Cada capacitación tuvo una duración de 4 semanas; se realizaron 205 trabajos prácticos, se entregaron 68 trabajos finales.

En el enlace siguiente se reúne la serie mencionada ttps://blogs. ead.unlp.edu.ar/discapacidadunlp/category/capacitaciones-virtuales/

\section{App UNLP AcceSíble}

(Relato realizado con el aporte de Evelina Díaz, María Innaro, Ivana Harari, lan Caballero y Martín Aparicio)

La Aplicación "UNLP AcceSíble", denominada de esta manera, en un juego de palabras que invitan a afirmar que nuestra universidad trabaja permanentemente en la creación de políticas de accesibilidad. Se trata de una aplicación para celulares que tiene el objetivo de informar y dar a conocer una universidad que intenta garantizar los derechos de las PCD, potenciando la autonomía de los estudiantes y el goce pleno de la vida universitaria.

Desde la Dirección adherimos a que el principio de la autonomía es básico en cualquier estructura democrática, ya que sin éste existe sustitución y opresión, (REDI, 2011), y es por ello que el principio de autonomía atraviesa nuestras políticas. 

mía es básico en cualquier estructura democrática, ya que sin éste existe sustitución y opresión, (REDI, 2011), y es por ello que el principio de autonomía atraviesa nuestras políticas.

La aplicación es el resultado de un trabajo colaborativo e interdisciplinario entre diferentes sectores de la comunidad universitaria: La Dirección, docentes y estudiantes de la Facultad de Informática, el grupo de Estudiantes por la Inclusión y la Accesibilidad - GEIA.

Compartimos la historia de este colectivo de estudiantes GEIA, ya que se reactivó en tiempo de pandemia. Con anterioridad estaban identificados como "Grupo de testeo" conformado por estudiantes con discapacidad que eran convocados cada vez que se necesitaba chequear la accesibilidad o conocer su opinión frente a alguna propuesta.

La App es la cristalización de nuestra forma de trabajo, construyendo políticas junto al colectivo de PCD, promoviendo su participación desde el inicio, adhiriendo al lema del movimiento de vida independiente "Nada sobre nosotros sin nosotros".

Entendemos que promover estas formas de participación, es una forma más de democratizar el acceso a la información y visibilizar la tarea que lleva a cabo esta Universidad generando políticas para eliminar las barreras que existen.

Es habitual que las políticas sean evaluadas una vez finalizado el proyecto, sin embargo, este proyecto tuvo una evaluación en proceso, contando con un diálogo permanente para comprobar la usabilidad y accesibilidad de la aplicación.

Los contenidos seleccionados para incorporar en la App son: Quiénes somos; Referentes de las 17 Unidades académicas donde presentan su área en un video accesible; Apoyos, Clínica jurídica; Espacio de consultas; Redes sociales; Contacto.

\section{Los contenidos seleccionados para incorporar en la App son: Quiénes somos; Referentes de las 17 Unidades académicas donde presentan su área en un video accesible; Apoyos, Clí- nica jurídica; Espacio de consultas; Redes sociales; Contacto.}

El equipo interviniente al momento de presentarlo explicó: "respecto al diseño, se optó por que sea simple, con información concisa y clara para facilitar la comprensión; se utilizaron etiquetas de texto alternativo para imágenes, íconos, botones y videos, una paleta de colores con alto nivel de contraste, cuerpos de tipografía adecuados para personas 
con discapacidad visual. Para el acceso a la información de las personas sordas y atendiendo a su demanda, se generó un sistema de pictogramas para el menú principal como correlato visual de los nombres de las secciones y los videos cuentan con subtitulado e intérprete de lengua de señas; se generaron elementos "clickeables" con un tamaño tal que permita su uso a personas con dificultades motrices. En tal sentido, tanto el diseño como la arquitectura de la aplicación cumplen con los estándares de accesibilidad del consorcio WCAG (Web Content Accessibility Guidelines) a fin de que las personas con discapacidad puedan percibir y comprender sus contenidos, así como navegar e interactuar sin dificultades. Una vez definido el diseño general, comenzó el proceso de desarrollo e implementación de la aplicación. Como validadores de accesibilidad se utilizaron las siguientes herramientas:

- Test de Accesibilidad de Google: permite analizar cada pantalla de la aplicación indicando si la misma cumple con los estándares básicos de accesibilidad mencionados en los requerimientos.

- Contrast Checker: herramienta online para lograr una paleta de colores accesible de manera rápida. Indica si los mismos generan un contraste accesible y sugiere colores alternativos o para combinar.

- TalkBack: es un lector de pantalla. Describe en voz alta las acciones y elementos del dispositivo.

Esta aplicación está en sintonía con el Art. 9 de la CDPD respecto a la accesibilidad, que establece:

"A fin de que las personas con discapacidad puedan vivir en forma independiente y participar plenamente en todos los aspectos de la vida, los Estados Partes adoptarán medidas pertinentes para asegurar el acceso de las personas con discapacidad, en igualdad de condiciones con las demás, al entorno físico, el transporte, la información y las comunicaciones, incluidos los sistemas y las tecnologías de la información y las comunicaciones, y a otros servicios e instalaciones abiertos al público o de uso público, tanto en zonas urbanas como rurales"

Compartimos la reflexión que hizo uno de los estudiantes de informática:

“Desde mi punto de vista, fue un gran desafío. Jamás había participado de un proyecto real con estas características, lo cual al principio me generó dudas sobre mi capacidad, pero terminé dándome cuenta de que sí y reafirmé que con un buen equipo de trabajo todo se hace más fácil. Adquirí mucho conocimiento en el proceso, sobre todo lo que no se aprende tanto en el ámbito facultativo como interactuar con distintos 
tipos de personas y perfiles, medios y formas de comunicación. También me sirvió mucho para comprender más las dificultades de las personas en situación de discapacidad, siempre hay mucho más de lo que uno ve desde afuera de cualquier problemática, y cuando uno interioriza descubre nuevas cosas y corrige pensamientos".

La aplicación se puede descargar en: https://play.google.com/store/ apps/details?id=ar.edu.unlp.inclusion

Al poco tiempo de haberla lanzado pudimos comprobar que fue un producto valioso, ya que se pusieron en contacto estudiantes de la UNLP, como así también de otras universidades, consultando y dando a conocer apoyos que requerían.

\section{Al poco tiempo de haberla lanzado pudimos comprobar que fue un producto valioso, ya que se pusieron en contacto es- tudiantes de la UNLP, como así también de otras universida- des, consultando y dando a conocer apoyos que requerían.}

Para ir concluyendo invitamos a ponerse en contacto a quienes estén interesados, no solo como lectores, sino que quieran pensar estrategias para accesibilizar su ámbito laboral.

Trabajar en clave de transversalidad interpela pero tambien invita a materializar la proyección de una universidad inclusiva, accesible, democrática y feminista en diálogo con cada una de las personas que habitan la Universidad y las áreas intervinientes. 


\section{BIBLIOGRAFIA}

Angelino, A; Rosato, A. et. al. (2009). El papel de la ideología de la normalidad en la producción de discapacidad. En Revista Ciencia, Docencia y Tecnología, № 39, 2009, noviembre de 2009, pp. 87-105. Recuperado de https://www.redalyc.org/pdf/145/14512426004.pdf

Convención sobre los derechos de las Personas con Discapacidad (2006). Recuperado de http://www.un.org/esa/socdev/enable/documents/tccconvs.pdf

Joly, E. (2011). ¿Qué les pasa a la ciencia, a la tecnología y a la universidad con relación a las personas con discapacidad? ¿Las ven, las escuchan, o son sordas y ciegas? Katz, S y Danel, P (compiladoras) Hacia una Universidad Accesible. Construcciones colectivas por la discapacidad Edulp. Buenos Aires. Argentina

Krause Arriagada, A; García Rodríguez, G; Katz, S; y Rodríguez Morales, S. Universitarios con discapacidad: realidades y desafíos en contexto de pandemia de la Red Interuniversitaria Latinoamericana y del Caribe sobre Discapacidad y Derechos Humanos. lesalc. Unesco. ESS. Vol. 33 Núm. 2 (2021). Recuperado de https://www.iesalc.unesco.org/ess/index.php/ess3/issue/view/46

REDI. El derecho a la autonomía de las personas con discapacidad como instrumento para la participación social, 2011. Recuperado de https://docplayer.es/18305693-El-derecho-a-la-autonomia-de-las-personas-con-discapacidad-como-instrumento-para-la-participacion-social.html 